\title{
Method for Identifying Representative Failures in Modular Products Through Field Application Data Analysis
}

\author{
Evandro CERQUEIRA $^{\mathrm{a}, 1}$, Rafael VOLTOLINI ${ }^{\mathrm{a}}$ and Milton BORSATO ${ }^{\mathrm{a}}$ \\ ${ }^{\mathrm{a}}$ Federal University of Technology - Paraná (UTFPR), Brazil
}

\begin{abstract}
Increasingly, manufacturing companies are focusing their efforts on exploring new markets. This new reality makes them strive for more efficient ways to offer their products at a lower cost and without losing their customization. As a result, the compromise between volume and customization (i.e. mass customization) is necessary and to support these product platforms have become a standard practice in the industry, especially the automotive one. However, another challenge arises with the use of platforms: the lack of an efficient way to develop product platforms that will bring a high level of customer satisfaction. The present work aims to develop a method capable of assisting global project groups for identifying representative failures in modules of product platforms and to set up product variations. It is intended to solve the problem of inefficient platform configuration for different markets, taking into account the specific application characteristics of each one. The methodological procedure is based on the Design Science Research (DSR) framework, according to which the work is carried out in six steps. The demonstration and evaluation steps of the solution were performed in the context of an automotive partner industry. The results show that is possible to use the method as a way to improve product platform configuration. The main contribution comes from the fact that the method performs a data analysis based on actual usage information under different product application conditions.
\end{abstract}

Keywords. Product Development, Product Platform, Platform Configuration, Mass Customization

\section{Introduction}

In the current context, companies have been looking for more efficient ways to serve the various markets in which they operate, with low cost of product development and production. With the increasingly globalized world, the newly created products need to meet the demands of different consumer markets. The key to success in a highly competitive manufacturing environment is the ability of companies to create and develop products that can adapt to customer needs [1]. As the number of markets served increases, companies need to develop more specific products. To address this issue, companies are looking for more efficient ways to lower their development costs. Considering this scenario, the product platforms were developed. One of the most cited references when it comes to product platforms is the article by [2], where the authors

\footnotetext{
${ }^{1}$ Corresponding Author. [evandrocerqueira@yahoo.com.br]
} 
define product platforms as the set of parameters, features and/or components that remain constant from product to product within a particular product family. Next to the product platform concept, we have the concept of modularity, which is of fundamental importance when it comes to component sharing. A module in a typical modular system has many internal connections between the internal elements of the module but has relatively few external connections to other modules [3].

One of the biggest challenges for companies is how to efficiently configure product platforms. They must be able to meet market demands, especially in terms of customer perceived quality, while bringing cost reduction to the company.

Requirements from different market applications can lead to complex product platforms. As a way to assist the Product Development Process (PDP) in configuring product platforms, the use of field information databases can be considered as a supporting process to identify modules that have representative field failures.

The objective of this work is to develop a method to support global R\&D groups to identify representative failures in the modules used in product platforms. It is considered specific application conditions of each market. Using the method, it will be possible to identify in which applications and product variants the modules have the highest failure rates. The project team can use this information to more efficiently configure product platforms. Once the developed method is presented, it is demonstrated in an industrial environment. Finally, the results and conclusions are presented.

\section{Product Platform Configuration Issues}

The research by [4] seeks to study the problem of modeling the structure of the product family. For this, the network analysis method was applied in the modeling of the product family structure. With this method, it was possible to balance between standardization and differentiation of the modular product platform. This method does not apply to configure the same product platform, but to model the structure of a product family. [5] were concerned with determining the right platform portfolio in which products from a product family are assembled. They created a model that can guide companies to develop a portfolio of lower-cost platforms, where cost is expressed in terms of the difference in technical attributes between the platform and the product.

The study by [6] develops a methodology that allows companies to determine the "optimal configuration" of a product, based on the similarities between variants of a new product and a product already offered by the company. The work contributes to providing a methodology that reduces the company's internal effort to differentiate products while meeting customer needs. In order to identify the appropriate point between several product variants and complexity, [1] develops a methodological framework for generating all possible product configurations based on the number and types of components or modules available on the assembly line. Thus, complexity has been categorized into levels to scale products in terms of their complexity.

Regarding product complexity, [7] study the relationship between product variety and production quality in the context of the truck industry. This relationship is analyzed through process planning, cycle time and perceived production complexity in the company's manufacturing environment. When it comes to customized products, the ability of production to absorb the assembly of the various product variants well-led [8] 
to develop a study on the modular assembly line. In the study, the authors focus on the final product assembly, where the traditional series assembly line is unable to meet the high production demand for different products.

Given the various studies found on the subject, this paper aims to answer the following question: Could it be possible to configure a product platform to be used in different consumer markets, considering specific market application characteristics, and using R\&D groups geographically separated?

\section{MPCDATA - Method for Platform Configuration Using Data Analysis.}

The construction of the method began with the observation of failure occurrences in the modules of a product platform. The goal was to understand where the failures occurred and what were the most important factors that caused the failures. It was used a database that could show the history of failures. After some research and information exchange within the product development area of a heavy-duty vehicle company, we found a database that could be used. The database in question is generated through dealers authorized to perform repair services on their vehicles. Through the history of failures, it was observed that there is a very important factor that is related to the impact of different applications on module failure occurrences. It was found that a module could perform depending on the product application.

Through interviews with professionals from the automotive industry, it was found that there is no structured way to correlate different applications with product platform performance in order to identify the modules that presents low performance. Thus, it was necessary to develop a structured way to identify which applications the modules had the most failures. Thus, later use this information to assist the project team in effective engineering actions.

The next step was to write the method. Since the method concerns a procedure (step by step), each of the activities has been carefully described to arrive at an output that meets the overall goal. Once this step was completed, a preliminary test of the method application was performed. The goal was to understand if there were gaps in information and content. After some rounds of corrections and adjustments, a final form for the proposed method was reached.

The method has been separated into 7 steps to aid your understanding. Table 1 shows a description of each of the steps.

Table 1. Seven steps of method.

\begin{tabular}{cl}
\hline Step & \multicolumn{1}{c}{ Description } \\
\hline 1 & Database loading \\
2 & Setting Search Parameters \\
3 & Verification of results and identification of components \\
4 & Use DFMEA criteria for fault occurrence \\
5 & Application classification and occurrence checking \\
6 & Using DFMEA Criteria for Keyword Severity and Search \\
7 & Engineering Action Strategy \\
\hline
\end{tabular}

By the end of the method, PDP will have identified the applications that most contribute to the occurrence of failures. Other important information is the modules that are most impacted in terms of failure occurrence for each of the applications. 
The method contributes to a better configuration of the product platform, in terms of choosing which modules will be a permanent part of the platform and which ones can be developed by local engineering groups. The following is each step in detail.

The first step is to access the database. The database, generated through work orders, presents various information about the product and the faults found. At the end of the first step, the database is loaded and product engineering can access it. The important thing is to have a reliable field complaint database. Accessibility for this database should also be taken into account, it should be easily accessible to product engineers. This will ensure greater independence of the method user within the PDP.

In step 2 the data analysis work begins. For this, it is important to define which filters to apply to the database. Filters will help direct the search for failed components. Starting with choosing the platform, the user needs to define which platform he wants to perform data analysis on. Next, the user chooses the product variants within the product family he wants to analyze. The next filter is the product platform module choice. The next activity is to define the other relevant modules. This step is intended to give the user the freedom to choose other modules or components that they consider relevant to understanding the faults. The last search filter concerns setting the time period. In this activity, the user needs to choose the time period of interest to filter the data.

Step 3 begins with the results verification activity. This is a step where the user needs to check if after appling filters failures will occur for any component. After this activity, two options are possible. The first option is that no module component has a failure $\log$ within the database. Thus, it can be concluded that the platform is configured correctly. If any faulty components appear, the method follows option 2 . In this option, there is at least one failure record for some components. Thus, the activity output is a list of components that have failed in the field. This list serves as input data for the next activity, called Identify Components. In this activity, failed components are identified.

In step 4, the first activity aims to establish a fault occurrence criterion. From this criterion, the product development engineering team can determine from which level of failure occurrence it is necessary to implement some modification in the module under study. To do this, you must use the Failure Mode and Effect Analysis (FMEA) method. The rating value set was 8 . The reason for choosing this rating value stems from the fact that the failure that is likely or uncertain is connected to the new application. According to the failure assessment criteria established above, there are two options. First, when all failed identified components are rated less than 8, then the method has reached an output called No Engineering Actions. The second option occurs when any of the components has a failure rating value equal to or greater than 8 . In this case, the uncertainty arising from a new application generates a need to proceed with a more detailed data analysis.

Step 5 begins by classifying product applications. In this activity, it is need to look for ways to classify field applications. The collection of this information should be the responsibility of the user of the method, taking into account his knowledge of the product, and the redemption of this information may vary according to the available database. Once the product application classification activity has been completed, the activity of checking for application failures begins. During this activity, the user must make a careful analysis of the failure occurrences for each of the applications. Finally, the method proceeds to the activity called Check for Failures by Product Variant. By combining information from the previous activity with the activity of including product 
variants in data analysis, the amount of possible information to be analyzed further expands.

Step 6 begins with reading the failures reports. Databases generally have the information written by the service technicians about what caused the failure. Reading activity requires knowledge of the product and its possible failure modes. This is because often the service technicians does not fully describe what caused the failure. The established way to describe the fault more briefly is to remove keywords from the text from the fault records. The purpose of this activity is to find the keyword that most characterizes the failure. Once all the keywords have been identified, a severity level must now be set for the failures recorded. FMEA was used to establish a failure severity classification criterion. During this activity, the user must establish the corresponding failure rate for each record. The activity of checking the most used keywords per application aims to identify a correlation between failure modes and application types. Similarly, the activity of verifying the severity of failure by application, it is possible to combine the results of the severity indexes previously raised for each application of the product.

The last step of the method is the grouping of all information and set the most appropriated strategy definitions for engineering actions. It starts with the activity of determining which product variants need action. By combining the variant list with its applications, keywords, and severity index, the information table will be complete. During the activity of determining the engineering strategy, the product engineer will analyze all application information from the field, and direct remediation actions according to the information gathered during the data analysis process.

During the process of defining module modification strategies, some engineering action options should be considered. The method highlight 4 possible actions:

1. Strengthen or improve the current solution;

2. Choose another platform module to replace the current module;

3. Develop a new module only for the most faulty product variants;

4. Develop a new module for all product variants.

In the first option, the engineer will seek to improve the performance of the module that is experiencing high levels of field failure. The second option assumes that other modules are belonging to the platform that could replace the defective module. The third option should be considered if it is not possible to completely replace the underperforming module. The last engineering strategy option concerns the development of a new module for all product variants.

\section{Real Case of Method Application in the Automotive Industry}

In this section, a real application of the developed method will be demonstrated. The demonstration took place in an industrial setting. The objective is to verify if the method is capable of meeting the proposed objective.

To demonstrate the method, a specific module for data analysis was chosen. Among the modules, one was chosen that had a very specific application characteristic. It is the only module available for all product variants of the chosen platform. There is no other module that can be used to perform the same function. In all applications, this is the only module to be used. Its function is to prevent debris from being thrown by 
the wheels. It is a system used on most heavy-duty vehicles for external protection. It is called a mudguard. Figure 1 shows a detailed image of the module.

The first step of the method has started. The database generated by the dealers workshops containing the field complaint records was loaded. This database is initially accessed through the Microsoft Excel program.

In step 2 the search parameters were defined, where the data filtering is performed later. The platform most used by the company was chosen, where the highest complaint rates in the field are registered. In order to choose which product variants to analyze, it was decided to chose all of them. The additional module chosen was the suspension module. This module will be used to classify applications. Finally, a period of 6 months was chosen for the analysis.

Step 3 started with verifying the results. With this first analysis, it was possible to identify the components of the chosen module that presented higher customer complaint rates.

With the identified components, the method proceeded to step 4 . In the first activity, the DFMEA criterion was applied to survey the failure occurrences for each of the components. Thus, only the component $\mathrm{H}$ of the module continued to be analyzed by the method, where it presented 103 fault records. The rest of the components fall under the criterion option less than 8, where there is no engineering action.

Step 5 was started with a survey of the applications. Considering the heavy vehicles, the characterization of applications is strongly related to road conditions and vehicle load capacity. Thus, 3 different types of applications were classified according to the type of suspension module: Light, medium and severe.

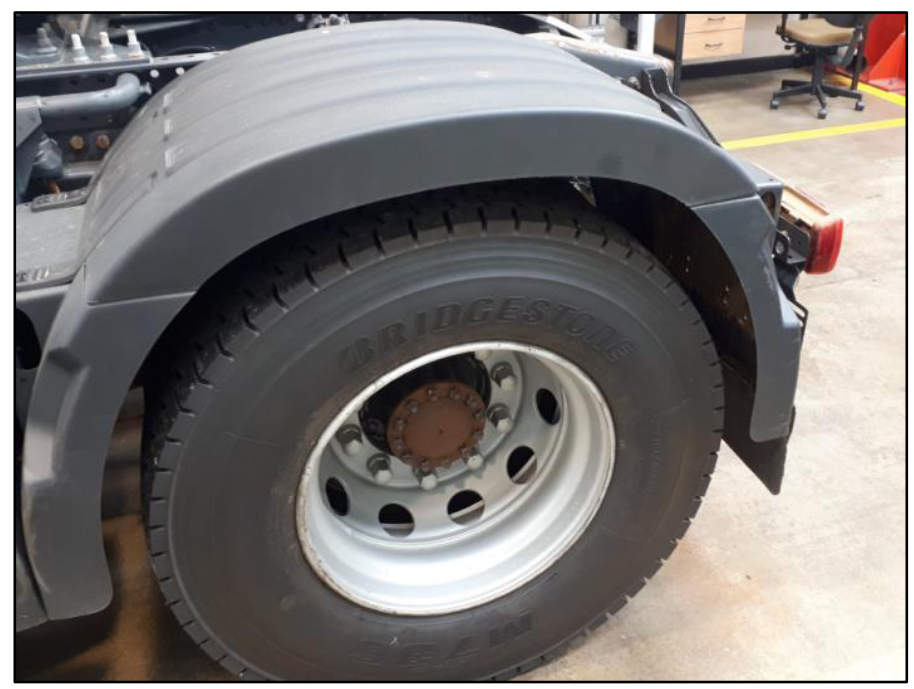

Figure 1. Module used in method demonstration.

From the classification of applications, it was possible to perform the activity of checking the occurrence of failures by the application. Likewise, the number of failure occurrence for each product variant was listed, considering the different applications. It can be ckecked in the table 2 . 
Table 2. Failures occurence per product variant and application

\begin{tabular}{cccc}
\hline Component & Application & Product Variant & Failures Occurence \\
\hline \multirow{3}{*}{ Component H } & Light & Variant A & 44 \\
& & Variant E & 1 \\
& \multirow{2}{*}{ Medium } & Variant B & 57 \\
& & Variant C & 1 \\
\hline
\end{tabular}

Looking at the table above, we can conclude that variants $\mathrm{A}$ and $\mathrm{B}$ are the most influential in the occurrence of faults for component $H$. Thus, these two variants can be used for future component correction validation actions.

Step 6 began by reading each of the 103 failures logs. The first activity was the filtering of the keywords that determine the failure and the second concerns the application of the failure severity rating criterion, according to DFMEA. To extract the keyword from each of the records, it was identified the word that most characterized the failure that occurred. The keywords that most characterized the failures for component $\mathrm{H}$ were breaking and tear respectively. Regarding the severity rating, 6 presented the highest occurrence in the failure records for component $\mathrm{H}$, being well distributed between the light and medium applications.

The first activity of step 7 was to analyze for which product variants, an engineering strategy should be defined for component H. For this purpose, all the information gathered up to that moment during the data analysis were grouped in a single frame. Table 3 shows a summary of all the information collected during data analysis.

Table 3. Summary of information gathered from data analysis

\begin{tabular}{|c|c|c|c|c|c|c|}
\hline \multirow[b]{2}{*}{ Component } & \multirow[b]{2}{*}{$\begin{array}{l}\text { Severity } \\
\text { Rating }\end{array}$} & \multirow[b]{2}{*}{ Keywords } & \multicolumn{4}{|c|}{ Product Variant/Aplication } \\
\hline & & & $\begin{array}{c}\text { Variant A / } \\
\text { Light }\end{array}$ & $\begin{array}{c}\text { Variant B / } \\
\text { Medium }\end{array}$ & $\begin{array}{l}\text { Variant C / } \\
\text { Medium }\end{array}$ & $\begin{array}{c}\text { Variant E } \\
\text { / Light }\end{array}$ \\
\hline \multirow{14}{*}{ Component $\mathrm{H}$} & \multirow{5}{*}{3} & Loss & & 2 & & \\
\hline & & Breaking & & 3 & & \\
\hline & & Crack & 1 & 1 & & \\
\hline & & Tear & 7 & 8 & & \\
\hline & & Snap & 4 & 8 & & \\
\hline & \multirow{6}{*}{6} & Fracture & & 1 & & \\
\hline & & Broken & & 3 & & \\
\hline & & Loss & 4 & & & \\
\hline & & Breaking & 5 & 10 & & \\
\hline & & Tear & 7 & 8 & 1 & 1 \\
\hline & & Snap & 14 & 7 & & \\
\hline & \multirow{3}{*}{8} & Breaking & 1 & & & \\
\hline & & Tear & & 2 & & \\
\hline & & Snap & 1 & 4 & & \\
\hline
\end{tabular}

An engineering strategy was defined replacing component $\mathrm{H}$ with a stronger one, where better quality results are expected. However, if we consider the main fender module used in the demonstration, the engineering action taken corresponds to option 1 which is to reinforce or improve the current solution. Finally, the replacement was performed based on the data analysis presented here. With this, the demonstration of the method was completed. In the end, it was possible to identify in which applications the module presented the highest failure occurrence rates. Also, it is possible to identify the product variants most impacted by the different applications. 


\section{Discussion of results}

Table 3 shows a summary of what the final information table combined by the product variant looked like. For component $\mathrm{H}$, product variant $\mathrm{B}$ has the highest number of failures, followed by variant A.

The breaking and tear keywords are the ones that most appeared in the records characterizing failure modes. Severity index 6 is the most commonly considered failure severity criterion. As already shown, the applications considered as light and medium were the ones with the most failure occurrence.

As a focus of engineering action to improve field performance of component $\mathrm{H}$, product variants $\mathrm{B}$ and $\mathrm{A}$ should be considered respectively. For example, these two variants could be used as a means of physically validating a new $\mathrm{H}$ component.

\section{Conclusion and future research opportunities}

Concerning diagnosing the efficiency of product platform configuration, the developed method has shown that it is feasible to use a database formed from field complaint records.

This database makes it more reliable to analyze the efficiency of the product platform configuration as it uses as a data analysis basis for the actual records of different markets and applications. The different applications and their impacts on each of the product variants is one of the most important information generated from the database. As mentioned, different applications influence the performance of platform modules. The method was able to use information from different product applications as a key factor to help the project team make strategic decisions on product improvement actions.

Another benefit of the method is related to using failure severity indices to quantify the failures reported by customers. Taking advantage of the same criteria used in DFMEA helped make the method easier to use. The use of a widespread industry standard also helps efficiency as it makes the method faster to understand. The method crosses the severity index data and different applications, providing a better understanding of where failures are occurring and what impact on the customer.

Some future research opportunities have been raised. There is a possibility to automate the method. For this to be done more efficiently, it is necessary to develop a computer program that can perform the entire process. Another opportunity is to implement a failure cost factor. It is data that can also be redeemed in the database. Although the work presented here is not focused on the cost of failure, it is information that can be incremented in the method.

\section{References}

[1] V. Modrak, D. Marton, and S. Bednar, The influence of mass customization strategy on configuration complexity of assembly systems, Procedia CIRP, 2015, vol. 33, pp. 538-543.

[2] Meyer MH, Lehnerd AP, The power of product platform: building value and cost leadership. Free Press, New York, 1997.

[3] K. Sinha and E. S. Suh, Pareto-optimization of complex system architecture for structural complexity and modularity, Research in Engineering Design, 2018, vol. 29, pp. 123-141, 2018. 
[4] B. Fan, G. Qi, X. Hu, and T. Yu, A network methodology for structure-oriented modular product platform planning, Journal of Intelligent Manufacturing, 2015, vol. 26, pp. 553-570.

[5] M. Van den Broeke, R. Boute, B. Cardoen, and B. Samii, An efficient solution method to design the cost-minimizing platform portfolio, European Journal of Operational Research, 2017, vol. 259, pp. 236-250.

[6] G. Schuh, S. Rudolf, and M. Riesener, Similarity-based product configuration, Procedia CIRP, 2014, vol. 17 , pp. $290-295$.

[7] P. E. C. Johansson, S. Mattsson, L. Moestam, and Å. Fast-Berglund, Multi-variant Truck Production Product Variety and its Impact on Production Quality in Manual Assembly, Procedia CIRP, 2016, vol. 54, pp. 245-250.

[8] W. Ren, Y. Guan, and Y. Hu, ScienceDirect Research on assembly module partition for flexible production in mass customization, Procedia CIRP, 2018, vol. 00, pp. 744-749. 